\title{
Concurrent Non-Malleable Zero Knowledge with Adaptive Inputs
}

\author{
Huijia Lin ${ }^{\star}$ and Rafael Pass ${ }^{\star \star}$ \\ Cornell University \\ $\{$ huijia, rafael\}@cs. cornell.edu
}

\begin{abstract}
Concurrent non-malleable zero-knowledge $(\mathcal{C N M \mathcal { K }})$ considers the concurrent execution of zero-knowledge protocols in a setting where the attacker can simultaneously corrupt multiple provers and ver-

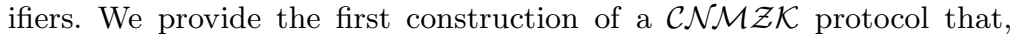
without any trusted set-up, remains secure even if the attacker may adaptively select the statements to receive proofs of; previous works only handle scenarios where the statements are fixed at the beginning of the execution, or chosen adaptively from a restricted set of statements.
\end{abstract}

\section{Introduction}

Zero-knowledge $(\mathcal{Z K})$ interactive proofs GMR89 are fundamental constructs that allow the Prover to convince the Verifier of the validity of a mathematical statement $x \in L$, while providing zero additional knowledge to the Verifier. Concurrent $\mathcal{Z} \mathcal{K}$, first introduced and achieved by Dwork, Naor and Sahai [DNS04], considers the execution of zero-knowledge protocols in an asynchronous and concurrent setting. In this model, an adversary acts as verifiers in many concurrent executions of the zero-knowledge protocol, and launches a coordinated attack on multiple independent provers to gain knowledge. Non-malleable $\mathcal{Z} \mathcal{K}$, first introduced and achieved by Dolev, Dwork and Naor DDN00, also considers the concurrent execution of zero-knowledge protocols, but in a different manner. In this model, an adversary concurrently participates in only two executions, but plays different roles in the two executions; in the first execution (called the left execution), it acts as a verifier, whereas in the second execution (called the right execution) it acts as a prover. The notion of Concurrent Non-malleable $\mathcal{Z} \mathcal{K}$ $(\mathcal{C N M Z K})$ considers both of the above attacks; the adversary may participate in an unbounded number of concurrent executions, playing the role of a prover in some, and the role of a verifier in others. Despite the generality of such an attacks scenario, this notion of security seems most appropriate for modeling the execution of cryptographic protocols in open networks, such as the Internet.

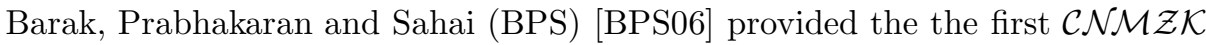

\footnotetext{
${ }^{\star}$ Lin is supported by a Microsoft Research Fellowship.

${ }^{\star}$ Pass is supported in part by a Microsoft New Faculty Fellowship, NSF CAREER Award CCF-0746990, AFOSR Award F56-8414, BSF Grant 2006317 and I3P grant 2006CS-001-0000001-02.
} 
argument for $\mathcal{N} \mathcal{P}$ in the plain model (i.e., without any set-up assumptions); see also the more efficient instantiation of Ostrovsky, Pandey and Visconti OPV10. More recently, Lin, Pass, Tseng and Venkitasubramaniam (LPTV) LPTV10. provided a somewhat different approach to constructing $\mathcal{C} \mathcal{N} \mathcal{M} \mathcal{K}$ protocols, improving the round-complexity of the BPS construction, as well as providing a construction of a $\mathcal{C N M Z K}$ proof.

Adaptive Inputs Selection. All the above-mentioned feasibility results for $\mathcal{C N M Z K}$, however, consider a quite restricted form of input selection: More precisely, whereas the attacker is allowed to adaptively select the statements it gives proofs of (on the right), the statements to receive proofs of (on the left) are assumed to be fixed before the execution begins.

Indeed, there is a sense in which this is necessary: as argued by Lindell [Lin03], if we consider a scenario where the left statement are chosen adaptively by an "environment" (think of this as some other arbitrary protocol running in the network), then the notion of $\mathcal{C N M \mathcal { K }}$ collapses down to the notion of Universally Composable $\mathcal{Z} \mathcal{K}$ Can01, which is known to be unachievable without set-up [CKL03.

We here focus on the simpler case of just "self-composition": that is, we only consider the security of the $\mathcal{Z K}$ protocols (and thus we do not allow them to interact with other protocols in the network; this is similar to the original setting studied in the context of Concurrent $\mathcal{Z K}$ ). Yet, we want to capture a notion of security where also the statements in the left executions are adaptively chosen. The natural way to do this is to (just as in the definition of security of signature schemes GMR89]) allow the attacker to adaptively select the statements it wants to hear proofs of on the left (as well as the statements it gives proofs of on the right); additionally we must restrict the attacker to only ask to hear proofs of statements that are true (or else we can never expect the conversation with the provers to be $\mathcal{Z} \mathcal{K}$-if the statement is false, then the prover on the left will not be able to provide a proof, which thus reveals information). Once we consider such adaptive instance selection, we also need to specify where the witnesses for the left interaction come from; to make the definition as general as possible, we consider an arbitrary (potentially unbounded) witnesses selecting machine that receives as input the views of all parties (i.e., the honest prover, the honest verifiers, and the adversary) and outputs a witness $w$ for any statement $x$ requested by the adversary.

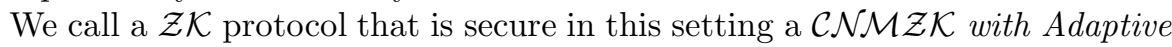

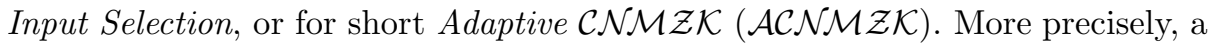
$\mathcal{Z} \mathcal{K}$ protocol is $\mathcal{A C N \mathcal { M Z } K}$ if for every adversary $A$, there exists a computationally efficient simulator-extractor that can simulate both the left and the right interactions for $A$, while outputting a witness for every statement proved by the adversary in the right interactions. Our main result is the construction of an

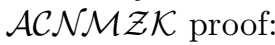

Theorem 1. Assume the existence of collision-resistant hash functions. Then there exists a $\omega\left(\log ^{2} n\right)$-round concurrent non-malleable zero-knowledge proof with adaptive input selection (and with a black-box simulator) for all of $\mathcal{N} \mathcal{P}$. 
Weaker Notions of Adaptive Input Selection. Our definition of adaptive input selection is strong in the sense that we allow the adversary to select any instance $x$ (as long as it is true), and somehow "magically" it receives a proof of this statement. Often, it suffices to restrict to adversaries that only are allowed to request statements $x$ for which there is an efficient way to recover a witness if having some auxiliary information. We may consider two ways of formalizing this.

- We may restrict the witness selecting machine to be a computationally bounded non-uniform algorithm that upon receiving a statement $x$ (and potentially also the view of the adversary), but not the view of the honest provers and verifiers, outputs a witness $w$. This models a scenario where the adversary is restricted to only requesting proofs of statements $x$ for which a witness can be efficiently computed using a "super witness". This is a natural extension of the fixed input scenario (again we have a witness - namely the "super witness" - that is fixed before the interaction) and indeed the result of BPS06, LPTV10, handle also such a notion of adaptive input selection.

- A less restrictive method is to simply restrict the witness selecting machine to be a computationally bounded non-uniform algorithm (which still receives the views of all parties). In particular, this allows us to model a scenario where the adversary may request a proof about earlier proofs (e.g., a proof that the prover has behaved honestly in an earlier proof) - in such a scenario, the honest prover may be able to efficiently find a witness, but there might not exists an efficient algorithm without having access to the prover's random tape. As far as we know, none of the earlier results handle even such a restricted notion of adaptive input selection.

Other Related Work. We mention that there are several works constructing $\mathcal{C} \mathcal{N} \mathcal{M Z K}$ protocols in various trusted set-up models. For instance, previous works $\mathrm{SCO}^{+} 01, \mathrm{CF} 01, \mathrm{DN02}$ ) provide constructions of Universally Composable $\mathcal{Z K}$ in the Common Reference String (CRS) model; these protocol are thus also

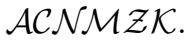

An interesting recent work by Yao, Yung and Zhao YYZ09] provide a construction of a $\mathcal{C N M \mathcal { K }}$ protocol in the Bare Public Key Model; their protocol is not $\mathrm{UC}$ secure but satisfies a notion CNMZK with adaptive inputs selection

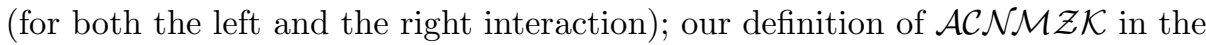
plain model is heavily inspired by their work.

Techniques. Our protocol is a close variant of the LPTV protocol; let us start by reviewing it. The protocol uses two main components. The first component is the notion of concurrently extractable commitments (CECom) introduced by Micciancio, Ong, Sahai, and Vadhan MOSV06]. Informally, values committed to using a CECom can be extracted by a rewinding simulator even in the concurrent setting. In our protocol (as in most concurrent $\mathcal{Z K}$ protocols), the verifier commits to a random trapdoor using CECom, so that our $\mathcal{Z} \mathcal{K}$ simulator may extract this trapdoor to perform simulation. The second component is the notion of robust non-malleable commitments (an extension due to Lin and Pass [LP09] 
of the notion of non-malleable commitments as defined by Dolev, Dwork, and Naor [DDN00]); roughly speaking these are non-malleable commitment schemes with an additional robustness property that makes them convenient to compose with other protocols.

The high-level idea behind the LPTV protocol (just as in the protocol of [BPS06]) is to start off with a preamble phase where the verifier uses a CECom to commit to a trapdoor; next in a commit phase, the prover commits to a witness of the proof statement using both a CECom and robust non-malleable commitments; and finally during a proof phase, the prover proves using a (standalone) $\mathcal{Z K}$ protocol that it has either committed to a valid witness, or a valid trapdoor in the commit phase. To prove security, LPTV provides a simulator that uses rewindings to extract out trapdoors (from the CECom in the preamble phase) to simulate the commit and proof phases of the left interactions, and uses rewindings again to extract the witnesses committed to by the adversary (from the CECom in the commit phase) on the right. The crux of the proof is then to show that even during the simulation, when the simulator commits to trapdoors (instead of real witnesses) in left interactions, the adversary still cannot commit to a trapdoor in right interactions, so that the values extracted out from the right interactions must be real witnesses. Very roughly speaking, this follows from the security guarantees of robust non-malleable commitments.

When considering adaptive input selection (for the left executions) a problem arises. First, proving indistinguishability of the simulation becomes problematic: in fact, getting a concurrent $\mathcal{Z} \mathcal{K}$ protocol with adaptive input selection is already non-trivial (we call it Adaptive Concurrent Zero-Knowledge $(\mathcal{A C Z K})$ ); our core technical contribution is to provide a solution to this problem. The reason for this is that proving indistinguishability of the simulation requires performing a hybrid argument, where we switch the witness used in the left interactions from the trapdoors (used by the simulator) to the real witness (used by the prover). More precisely, we consider a hybrid $H_{i}$, where the first $i$ left interactions are simulated using the trapdoors, and the later ones use the real witnesses. The problem is that the real witnesses might not be efficiently recoverable since the statements are chosen adaptively by the adversary (it is computed by a computationally unbounded witness-selecting machine); so the hybrid is not efficiently computable!

Our idea for circumventing this problem can be described as follows:

- First, we switch the order of the hybrids. We consider hybrids $H_{i}$ where the first $i$ left interactions are emulated using real witness and the later ones are simulated using trapdoors. The reason for doing this is that we can now non-uniformly fix the real witnesses of the first $i$ left interactions by hardcoding the "prefix" of hybrid $H_{i}$ before the $i^{\text {th }}$ left interaction; and then the remaining execution can be efficiently emulated using the real witnesses.

- But now the obstacle is that arguing indistinguishability of $H_{i}$ and $H_{i+1}$ becomes problematic. To show indistinguishability we need to show that simulating the $i^{\text {th }}$ left interaction using a real witness or trapdoor is indistinguishable (other interactions are simulated identically in the two hybrids). 
It seems that this should just follow from the hiding and $\mathcal{Z K}$ property of the commit and proof phases of the left interaction. However, the problem is that (when trying to extract the trapdoors of the latter left interactions), we might be rewindings the $i^{\text {th }}$ left interaction. Our way around this problem is to add more CECom to the preamble phase; the idea is to show that there exists some alternative simulator, that generates a statistically close distribution, but is able to avoid rewinding the messages in the commit and proof phases of the left interaction that we want to violate indistinguishability of.

To also complete the proof of non-malleability, a second (very related problem) arises: namely, we need to argue that the witness committed to by the adversary on the right are valid even in simulation; this is usually done through a hybrid argument as well and relies on the robust non-malleability of the commitment scheme used in the commit phase (instead of the hiding and $\mathcal{Z} \mathcal{K}$ properties). When doing this, we again run into the same problem as when showing indistinguishability of the simulation. Here the issue is that we need to ensure that the robust non-malleability property holds even under rewindings. We use the same idea to overcome this problem: as long as there are sufficiently many CECom in the preamble phase, we can describe an alternative simulator that produces a statistically close distribution without rewinding these commitments that we want to violate robust non-malleability of.

Overview. Section 2 contains the basic notations and definitions of $\mathcal{A C N \mathcal { Z } K}$ and other primitives. In Section 3 , we present our main result, a $\omega\left(\log ^{2} n\right)$-round $\mathcal{A C N M \mathcal { K }}$ proof system for all of $\mathcal{N} \mathcal{P}$, from collision resistant hash functions. In Section 4.2, we first focus on showing the $\mathcal{A C Z K}$ property of the protocol, which contains the main technical content of this paper; then in Section 5 we

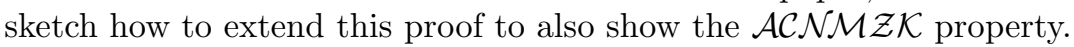

\section{Preliminaries}

Let $N$ denote the set of all positive integers. For any integer $n \in N$, let $[n]$ denote the set $\{1,2, \ldots, n\}$, and let $\{0,1\}^{n}$ denote the set of $n$-bit strings, and $\varepsilon$ the empty string. We assume familiarity with interactive Turing machines, interactive protocols, statistical/computational indistinguishability, notions of interactive proofs, zero-knowledge, (strong) witness-indistinguishability, and notions of statistically binding/hiding commitments. (See Gol01 for formal definitions.)

\subsection{Adaptive Concurrent Non-Malleable Zero-Knowledge}

Our definition of adaptive concurrent non-malleable zero-knowledge is very similar to that of concurrent non-malleable zero-knowledge from [BPS06] (which in turn closely follows the definition of simulation extractability of [PR05]), with the only difference that now the adversary is allowed to adaptively select the statements it receives proofs to, subject to that they are true statements.

Let $\langle P, V\rangle$ be an interactive proof for a language $L \in \mathcal{N} \mathcal{P}$ with witness relation $R_{L}$, and let $n$ be the security parameter. Consider a man-in-the-middle adversary 
$A$ that participates in many left and right interactions in which $m=m(n)$ proofs take place. In the left interactions, the adversary $A$ verifies the validity of statements $x_{1}, \ldots, x_{m}$ by interacting with an honest prover $P$, using identities $\mathrm{id}_{1}, \ldots, \mathrm{id}_{m}$. In the right interactions, $A$ proves the validity of statements $\tilde{x}_{1}, \ldots, \tilde{x}_{m}$ to an honest verifier $V$, using identities $\tilde{\mathrm{id}}_{1}, \ldots, \tilde{\mathrm{id}}_{m}$. Prior to the interactions, all parties in the system receives as common input the security parameter in unary $1^{n}$, and $A$ receives as auxiliary input $z \in\{0,1\}^{*}$. Furthermore, at the beginning of each left (respectively right) interaction, the adversary adaptively selects the statement $x_{i}$ (respectively $\left.\tilde{x}_{i}\right)$ and the identity $\mathrm{id}_{i}$ (respectively $\tilde{\mathrm{id}}_{i}$ ), with the only restriction that all the statements $x_{1}, \ldots, x_{m}$ chosen in the left interactions have to be true. Additionally in each left interaction, the prover $P$ receives as local input a witness $w_{i} \in R_{L}\left(x_{i}\right)$, chosen adaptively by a witness-selecting machine $M$. More specifically, $M$ is a (randomized) Turing machine that runs in exponential time, and whenever the adversary chooses a statement $x_{i}$ for a left interaction, $M$ on inputs the statement $x_{i}$ and the current view of all parties (including the adversary, provers, and receivers), picks a witness $w_{i} \in R_{L}\left(x_{i}\right)$ as the private input of the prover $P$. Let $\operatorname{view}_{A, M}(n, z)$ denote a random variable that describes the view of $A$ in the above experiment. Loosely speaking, an interactive proof is adaptive concurrent non-malleable zero-

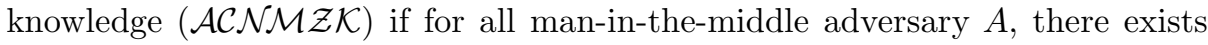
a probabilistic polynomial time machine (called the simulator-extractor) that can simulate both the left and the right interactions for $A$, while outputting a witness for every statement proved by the adversary in the right interactions.

Definition 1. An interactive proof $(P, V)$ for a language $L$ with witness relation $R_{L}$ is said to be adaptive concurrent non-malleable zero-knowledge if for every polynomial $m$, and every probabilistic polynomial-time man-in-the-middle adversary $A$ that participates in at most $m=m(n)$ concurrent executions, there exists a probabilistic polynomial time machine $S$, such that, for every input-selecting machine $M$ :

1. The following ensembles are computationally indistinguishable over $n \in N$

- $\left\{\operatorname{view}_{A, M}(n, z)\right\}_{n \in N, z \in\{0,1\}^{*}}$

$-\left\{S_{1}\left(1^{n}, z\right)\right\}_{n \in N, z \in\{0,1\}^{*}}$

where $S_{1}\left(1^{n}, z\right)$ denotes the first output of $S\left(1^{n}, z\right)$.

2. Let $z \in\{0,1\}^{*}$ and (view, $\boldsymbol{w}$ ) denote the output of $S\left(1^{n}, z\right)$. Let $\tilde{x}_{1}, \ldots, \tilde{x}_{m}$ be the statements of the right-interactions in view, and let $\mathrm{id}_{1}, \ldots, \mathrm{id}_{m}$ and $\tilde{\mathrm{id}}_{1}, \ldots, \tilde{\mathrm{id}}_{m}$ be the identities of the left-interactions and right-interactions in view. Then for every $i \in[m]$, if the $i^{\text {th }}$ right-interaction is accepting and $\tilde{\mathrm{id}}_{i} \neq \mathrm{id}_{j}, \boldsymbol{w}$ contains a witness $w_{i}$ such that $R_{L}\left(\tilde{x}_{i}, w_{i}\right)=1$.

We also consider concurrent $\mathcal{Z} \mathcal{K}$ with adaptive input selection. We say that an interactive proof $(P, V)$ is adaptive concurrent $\mathcal{Z} \mathcal{K}(\mathcal{A C} \mathcal{Z} \mathcal{K})$ if it satisfies the above definition with respect to adversaries that only receive proofs (and do not give proofs). 
Remark 1. As mentioned before, the security proof in BPS06, LPTV10 can be extended to show that their constructions of $\mathcal{C} \mathcal{N} \mathcal{M} \mathcal{Z}$ protocols satisfy a notion of $\mathcal{C} \mathcal{N} \mathcal{M} \mathcal{K}$ with "weak" adaptive input selection, where the adversary can only choose to hear proofs of statements for which a witness can be computed efficiently without knowing the random coins of the honest provers and verifiers. Formally, the witness-selecting machine is restricted to be computationally bounded (i.e., a non-uniform $\mathcal{P} \mathcal{P} \mathcal{T}$ ) and only receive as input a statement $x$ and the view of the adversary (but not the views of the left provers and right receivers.)

Remark 2. Universal Composability (UC) Can01 considers a more generalized form of adaptive input selection, where both the statements and witnesses are chosen adaptively by a separate entity called the "environment", which may communicate with the adversary in an arbitrary way. In contrast, our definition of $\mathcal{A C N M \mathcal { K }}$ only allows the witnesses to be selected by a separate entity, whereas the statements are chosen directly by the adversary. It has been shown that UC $\mathcal{Z K}$ is unachievable without set-up [CKL03. We mention that our construction actually satisfies a slight strengthening of the above definition of

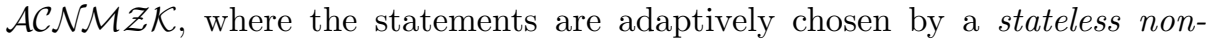
uniform $\mathcal{P} \mathcal{P} \mathcal{T}$ machine that both the adversary and the simulator have oracle accesses to. Such a notion bring us closer to the definition of UC $\mathcal{Z} \mathcal{K}$ - in essence, the difference is that in $\mathrm{UC} \mathcal{Z} \mathcal{K}$ the statement selecting machine is not necessarily stateless; we defer the details to the full version.

Non-Malleable Commitment Schemes. We recall the definition of nonmalleability from LPV08] (which builds upon the definition of [DDN00, PR05]). Let $\langle C, R\rangle$ be a tag-based statistically binding commitment scheme, and let $n \in N$ be a security parameter. Consider a man-in-the-middle adversary $A$ that, on auxiliary inputs $n$ and $z$, participates in one left and one right interaction simultaneously. In the left interaction, the man-in-the-middle adversary $A$ interacts with $C$, receiving a commitment to value $v$, using identity id of its choice. In the right interaction $A$ interacts with $R$ attempting to commit to a related value $\tilde{v}$, again using identity id of its choice. If the right commitment is invalid, or undefined, its value is set to $\perp$. Furthermore, if $\tilde{i d}=$ id, $\tilde{v}$ is also set to $\perp-$ i.e., a commitment where the adversary copies the identity of the left interaction is considered invalid. Let $\mathrm{nmc}_{\langle C, R\rangle}^{A}(v, z)$ denote a random variable that describes the value $\tilde{v}$ and the view of $A$, in the above experiment.

Definition 2. A statistically binding commitment scheme $\langle C, R\rangle$ is said to be non-malleable (with respect to itself) if for every polynomial $p(\cdot)$, and every probabilistic polynomial-time man-in-the-middle adversary $A$, the following ensembles are computationally indistinguishable.

$$
\begin{aligned}
& \left\{n m c_{\langle C, R\rangle}^{A}(v, z)\right\}_{n \in N, v \in\{0,1\}^{n}, v^{\prime} \in\{0,1\}^{n}, z \in\{0,1\}^{*}} \\
& \left\{n m c_{\langle C, R\rangle}^{A}\left(v^{\prime}, z\right)\right\}_{n \in N, v \in\{0,1\}^{n}, v^{\prime} \in\{0,1\}^{n}, z \in\{0,1\}^{*}}
\end{aligned}
$$


Non-Malleable Commitment Robust w.r.t. $\boldsymbol{k}$-Round Protocols. The notion of non-malleability w.r.t. arbitrary $k$-round protocols is introduced in LP09. Unlike traditional definitions of non-malleability, which only consider man-in-the middle adversaries that participate in two (or more) executions of the same protocol, non-malleability w.r.t. arbitrary protocols considers a class of adversaries that can participate in a left interaction of any arbitrary protocol. Below we recall the definition. Consider a one-many man-in-the-middle adversary $A$ that participates in one left interaction - communicating with a machine $B$ - and one right interaction - acting as a commiter using the commitment scheme $\langle C, R\rangle$. As in the standard definition of non-malleability, $A$ can adaptively choose the identity in the right interaction. We denote by $\mathrm{nmc}_{\langle C, R\rangle}^{B, A}(y, z)$ the random variable consisting of the view of $A(z)$ in a man-in-the-middle execution when communicating with $B(y)$ on the left and an honest receiver on the right, combined with the value $A(z)$ commits to on the right. Intuitively, we say that $\langle C, R\rangle$ is non-malleable w.r.t. $B$ if $\mathrm{nmc}_{\langle C, R\rangle}^{B, A}\left(y_{1}, z\right)$ and $\mathrm{nmc}_{\langle C, R\rangle}^{B, A}\left(y_{2}, z\right)$ are indistinguishable, whenever interactions with $B\left(y_{1}\right)$ and $B\left(y_{2}\right)$ cannot be distinguished.

Definition 3. Let $B$ be a probabilistic polynomial time machine. We say the statistically binding commitment scheme $\langle C, R\rangle$ is non-malleable w.r.t. $B$, if for every probabilistic polynomial-time man-in-the-middle adversary $A$, and every two sequences $\left\{y_{n}^{1}\right\}_{n \in N}$ and $\left\{y_{n}^{2}\right\}_{n \in N}$ such that, for all probabilistic polynomialtime machine $\tilde{A}$, it holds that

$$
\left\{\left\langle B\left(y_{n}^{1}\right), \tilde{A}(z)\right\rangle\left(1^{n}\right)\right\}_{n \in N, z \in\{0,1\}^{*}} \approx\left\{\left\langle B\left(y_{n}^{2}\right), \tilde{A}(z)\right\rangle\left(1^{n}\right)\right\}_{n \in N, z \in\{0,1\}^{*}}
$$

where $\langle B(y), \tilde{A}(z)\rangle\left(1^{n}\right)$ denotes the view of $\tilde{A}$ in interaction with $B$ on common input $1^{n}$, and private inputs $z$ and $y$ respectively, then it holds that:

$$
\left\{n m c_{\langle C, R\rangle}^{B, A}\left(y_{n}^{1}, z\right)\right\}_{n \in N, z \in\{0,1\}^{*}} \approx\left\{n m c_{\langle C, R\rangle}^{B, A}\left(y_{n}^{2}, z\right)\right\}_{n \in N, z \in\{0,1\}^{*}}
$$

We say that $\langle C, R\rangle$ is non-malleable w.r.t. $k$-round protocols if $\langle C, R\rangle$ is nonmalleable w.r.t. any $\mathcal{P} \mathcal{P} \mathcal{T}$ machine $B$ that interacts with the man-in-the-middle adversary in $k$ rounds. Below, we focus on commitment schemes that are nonmalleable w.r.t. itself and arbitrary $\ell(n)$-round protocols, where $\ell$ is a superlogarithmic function. We say that such a commitment scheme is robust w.r.t. $\ell(n)$-round protocols. The following result was shown in [LPV08].

Lemma 1 ([LPV08]). Let $\ell(n)$ be a super-logarithmic function. Then there exists a $O(\ell(n))$-round statistically binding commitment scheme that is robust w.r.t. $\ell(n)$-round protocols, assuming that one-way functions exist.

Concurrently Extractable Commitment Schemes. Micciancio, Ong, Sahai and Vadhan introduce and construct concurrently extractable commitment schemes, CECom, in MOSV06]. The commitment scheme is an abstraction of the 
preamble stage of the concurrent zero-knowledge protocol of [PRS02]. Informally, values committed by CECom can be extracted by a rewinding extractor (e.g., the zero-knowledge simulator of [KP01, PRS02, PTV08], even in the concurrent setting. In this work, we use the same construction as in [PRS02, MOSV06], but are unable to employ their analysis.

\section{An $\mathcal{A C N \mathcal { M Z K }}$ Proof}

In this section we construct an adaptive concurrent non-malleable zero-knowledge proof based on collision-resistant hash-functions. The construction is almost identical to the $\mathcal{C N M \mathcal { K }} \mathcal{K}$ proof system in [LPV10, except that, the verifier is asked to provide more CECom commitments to its trapdoor at the beginning of the protocol, which, in the proof, facilitates the simulator-extractor to extract the trapdoor while strategically avoiding rewinding certain messages.

Let $\ell(n)$ be any super logarithmic function. Our adaptive concurrent non-malleable zero-knowledge protocol, ACNMZKProof, employs several commitment protocols. Let $\mathrm{Com}_{s h}$ be a 2-round statistically hiding commitment (based on collision-resistant hash-functions), $\mathrm{Com}_{s b}$ be a 2-round statistically binding commitment (based on one-way functions), and NMCom be an $O(\ell(n))$ round statistically binding commitment scheme that is robust w.r.t. $\ell(n)$-round protocols (based on one-way functions).

Our protocol also employs $\ell(n)$-round statistically hiding (respectively statistically binding) concurrently-extractable commitment schemes, CECom sh $_{\text {(re- }}$ spectively $\mathrm{CECom}_{s b}$ ). These schemes are essentially instantiations of the PRS preamble PRS02, and can be constructed given $\mathrm{Com}_{s h}$ and $\mathrm{Com}_{s b}$. Below we repeat their definitions.

To commit a $n$-bit string $v$, the commiter chooses $n \times \ell(n)$ pairs of random $n$ bit strings $\left(\alpha_{i, j}^{0}, \alpha_{i, j}^{1}\right), i \in[n], j \in[\ell(n)]$, such that $\alpha_{i, j}^{0} \oplus \alpha_{i, j}^{1}=v$ for every $i$ and $j$. The sender then commits to $v$ and each of the $2 n \ell(n)$ strings in parallel using $\mathrm{Com}_{s h}$. This is followed by $\ell(n)$ rounds of interactions. In the $j^{\text {th }}$ interaction, the receiver sends a random $n$-bit challenge $b_{j}=b_{1, j} \ldots b_{n, j}$, and the commiter decommits the commitments of $\alpha_{1, j}^{b_{1, j}}, \ldots, \alpha_{n, j}^{b_{n, j}}$ according to the challenge.

A valid decommitment of $\mathrm{CECom}_{s h}$ requires the commiter to decommit all initial commitments under scheme $\mathrm{Com}_{s h}$ (i.e., reveal the randomness of the commitments), and that the decommitted values satisfy $\alpha_{i, j}^{0} \oplus \alpha_{i, j}^{1}=v$ for every $i$ and $j$.

A $\ell(n)$-round statistically binding concurrently-extractable commitment scheme, CECom sh $_{\text {, is defined analogously as CECom }}$, with the initial commitment $\mathrm{Com}_{s h}$ replaced by $\mathrm{Com}_{s b}$. Additionally, we say a transcript of CECom $s h$ is valid if there exists a valid decommitment.

We now describe ACNMZKProof, our adaptive concurrent non-malleable zeroknowledge protocol. Protocol ACNMZKProof for a language $L \in \mathcal{N} \mathcal{P}$ proceeds in six stages given a security parameter $n$, a common input statement $x \in\{0,1\}^{n}$, an identity id, and a private input $w \in R_{L}(x)$ to the Prover. 
Stage 1: The Verifier chooses a random string $r \in\{0,1\}^{n}$ and commits to $r$ using $k(n)+1$ invocations of CECom $s h$, where $k(n)$ is the number of rounds in Stage 2-6 of the protocol; $r$ is called the "fake witness".

Stage 2: The Prover commits to the witness $w$ using $\mathrm{CECom}_{s b}$.

Stage 3: The Prover commits to the witness $w$ using NMCom with identity id.

Stage 4: The Prover commits to the witness $w$ using NMCom with identity id, again.

Stage 5: The Verifier decommits the Stage 1 commitment to value $r^{\prime}$.

Stage 6: The Prover using a $\omega(1)$-round $\mathcal{Z K}$ proof (e.g., Blu86]), proves that the commitments in Stages 2, 3 and 4 all commit to the same value $\tilde{w}$ (with identity id), and that either $\tilde{w} \in R_{L}(x)$ or $\tilde{w}=r^{\prime}$.

A formal description of the protocol can be found in Figure 1 .

\section{Protocol ACNMZKProof}

Common Input: an instance $x$ of a language $L$ with witness relation $R_{L}$, an identifier id, and a security parameter $n$.

Auxiliary Input for Prover: a witness $w$, such that $(x, w) \in R_{L}(x)$.

Stage 1:

$\mathrm{V}$ uniformly chooses $r \in\{0,1\}^{n}$ (the "fake witness").

$\mathrm{V}$ commits to $r$ using $k+1$ invocations of the protocol CECom sh $_{\text {, where }}$ $k$ is the number of rounds in Stage 2-6 of the protocol. Let $\mathcal{T}_{1}$ be the commitment transcript.

\section{Stage 2:}

$\mathrm{P}$ commits to $w$ using protocol $\mathrm{CECom}_{s b}$. Let $\mathcal{T}_{2}$ be the commitment transcript.

\section{Stage 3:}

$\mathrm{P}$ commits to $w$ using protocol NMCom and identity id. Let $\mathcal{T}_{3}$ be the commitment transcript.

\section{Stage 4:}

$\mathrm{P}$ commits to $w$ using protocol NMCom and identity id. Let $\mathcal{T}_{4}$ be the commitment transcript.

\section{Stage 5:}

$\mathrm{V}$ decommits $\mathcal{T}_{1}$ to value $r ; \mathrm{P}$ aborts if no valid decommitment is given.

\section{Stage 6:}

$\mathrm{P} \leftrightarrow \mathrm{V}$ : a $\omega(1)$-round $\mathcal{Z} \mathcal{K}$ proof [Blu86] of the statement: There exists $\tilde{w}$ such that

- $\tilde{w}$ is a valid decommitment of $\mathcal{T}_{2}$,

- and $\tilde{w}$ is a valid decommitment of $\mathcal{T}_{3}$ and $\mathcal{T}_{4}$ under identity id, - and $\tilde{w} \in R_{L}(x)$ or $\tilde{w}=r$.

Fig. 1. An Adaptive Concurrent Non-Malleable $\mathcal{Z K}$ Proof for $\mathcal{N} \mathcal{P}$ 
On Round Complexity: Since the protocol NMCom has $O(\ell(n))$ rounds, we have that $k(n)=O(\ell(n))$. Therefore, the round complexity of the protocol ACNMZKProof is $O\left(\ell^{2}(n)\right)=\omega\left(\log ^{2} n\right)$.

The above protocol is an extension of the Goldreich-Kahan protocol [GK96]. Completeness and Soundness follows using stand techniques; since the protocol is essentially the same as the $\mathcal{C} \mathcal{N} \mathcal{M Z K}$ protocol in LPTV10] (except that Stage 1 now contains many CECom's), we refer the reader to [LPTV10] for more details.

\section{Proof of Security}

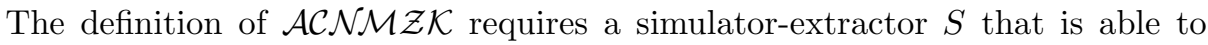
simulate the view of a man-in-the-middle adversary $A$ (including both left and right interactions), while simultaneously extracting the witnesses to statements proved in the right interactions. We describe the construction of our simulator in Section 4.1, show that it is a correct $\mathcal{A C Z \mathcal { K }}$ simulator in Section 4.2, and extend

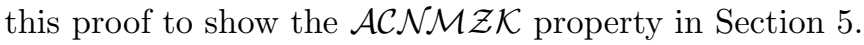

\subsection{Our Simulator-Extractor}

Our simulator-extractor, $S$, is almost identical to the simulator extractor of the $\mathcal{C} \mathcal{N} \mathcal{M K}$ protocol in [LPTV10, except that now, given more $\mathrm{CECom}_{s h}$ 's in Stage 1 of the protocol, $S$ tries to extract a "fake" witness from every CECom $s h$ from the adversary in the left interactions, and aborts if the extraction fails for any of the commitment or the extracted value does not equal to the value that the adversary decommitment to later. Roughly speaking, $S$ follows this strategy:

Simulating the view of the right interactions. $S$ simply follows the honest verifier strategy.

Simulating the view of the left interactions. In each protocol execution, $S$ first extracts the "fake witness" $r$ from the $k(n)+1$ CECom $_{s h}$ 's committed by $A$ in Stage 1, then commits to $r$ in Stage 2, 3, and 4, and finally simulates the $\mathcal{Z} \mathcal{K}$ proof using $r$ as a witness in Stage 6.

Extracting the witnesses. In each right interaction that completes success-

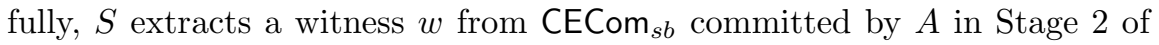
the protocol.

Thus, the main task of $S$ is to extract the values committed by $A$, using CECom, in Stage 1 and 2 of the protocol. This is done by rewinding $A$ during each CECom. To that end, we employ the "lazy KP" simulator of [PTV08, an oblivious simulator that is nearly identical to the Killian-Petrank (KP) simulator KP01. We also follow the analysis of [PTV08], which is in turn based on the analysis of [PRS02].

On a very high-level, $S$ attempts to simulate the view of $A$ (with "fake witnesses") in one continuous, straight-line manner (so as to not skew the output distribution); this is aided by numerous auxiliary rewinds that allows $S$ to extract the "fake witnesses" in time. As implied by our simulation strategy, the 
view of $A$ generated by $S$ depends on the extracted "fake witnesses", but is otherwise independent of the interaction in auxiliary rewinds. (The simulator $S$

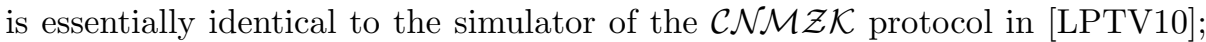
we refer the reader to [LPTV10] for a more detailed description.)

It is useful to know that $S$ may abort in two manners. At the end of a CECom, if $S$ is unable to extract the committed value (the rewinds were unhelpful), $S$ outputs $\perp_{\text {ext }}$. Or, in Stage 5 of a left interaction, if $A$ decommits its Stage 1 CECom $_{s h}$ 's to a value that is different from any of the $k(n)+1$ extracted values, $S$ outputs $\perp_{\text {bind }}$. Conversely if $S$ does not abort, then it must have extracted the committed value from every Stage $1 \mathrm{CECom}_{s h}$ that it has encountered, and $A$ must decommit to the extracted values (if $A$ decommits at all). The following claim bounds the abort probability of $S$.

Claim 2. $S$ outputs $\perp_{\text {ext }}$ and $\perp_{\text {bind }}$ with negligible probability.

The proof is identical to the proof of Claim 2 in LPTV10, which in turn follow directly from the analysis of [PTV08] in the setting of concurrent $\mathcal{Z K}$; we refer the reader to [LPTV10] for a formal proof.

\subsection{Proof of $\mathcal{A C Z K}$}

We first show that $S$ is a valid $\mathcal{A C Z K}$ simulator for the protocol ACNMZKProof, that is, the view generated by $S$ is indistinguishable from the real view of $A$.

Lemma 3. For every witness-selecting machine $M$, the following ensembles are computationally indistinguishable over $n \in N$ :

$$
\begin{array}{r}
\left\{S_{1}\left(1^{n}, z\right)\right\}_{n \in N, z \in\{0,1\}^{*}} \\
\left\{\operatorname{view}_{A, M}\left(1^{n}, z\right)\right\}_{n \in N, z \in\{0,1\}^{*}}
\end{array}
$$

To show Lemma 3, we introduce a series of hybrid simulators; the same hy-

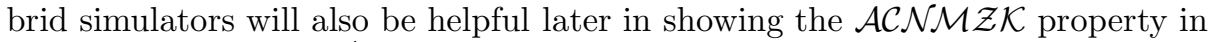
Section [5. Hybrids hyb ${ }^{i}, 0 \leq i \leq m+1$ proceed in three steps.

Real Execution Phase: Run the honest man-in-the-middle execution with $A$ until the $i^{\text {th }}$ left interaction starts: in left iteration $j<i$, run the witnessselecting machine $M$ (on input the statement $x_{j}$ of this interaction, and the current view of the adversary, prover and verifier) to compute a valid witnesses $w_{j}$ and execute the honest prover strategy. Note that the Real Execution Phase may take exponential time. Let $\mathcal{V}_{A}$ be the view of $A$, and $\mathcal{V}_{P}, \mathcal{V}_{V}$ the view of the prover and verifier produced in this phase.

Simulation Phase: Feed $A$ with $\mathcal{V}_{A}$. Run the following simulation strategy with $A$ to complete the partial execution defined by $\left(\mathcal{V}_{A}, \mathcal{V}_{P}, \mathcal{V}_{V}\right)$.

- For every right interaction, emulate the interaction by following the honest verifier strategy from $\mathcal{V}_{V}$.

- For left interaction $j<i$, emulate the interaction by following the honest prover strategy from $\mathcal{V}_{P}$. 
- For left interaction $j \geq i$, simulate the interaction using a "fake" witness, as $S$ does.

Formally, this simulation strategy can be implemented as follows: construct another machine $A^{\prime}$ that internally incorporates $A$ and simulates the first $i-1$ left and all right interactions for $A$ honestly from $\mathcal{V}_{P}$ and $\mathcal{V}_{V}$, and forwards the rest $m-i+1$ left interactions externally. Then simply run $S$ on $A^{\prime}$ and outputs the embedded view of $A$ in the view of $A^{\prime}$ produced by $S$.

Output Phase: Output $\perp_{e x t}$ or $\perp_{\text {bind }}$ if $S$ returns $\perp_{e x t}$ or $\perp_{\text {bind }}$; otherwise, output the view $\mathcal{V}$ of $A$ embedded in the view of $A^{\prime}$ produced by $S$.

We also define hybrids hyb ${ }_{+}^{i}$ that proceed identically to hyb ${ }^{i}$ except that, in the Simulation Phase, the $i^{\text {th }}$ left interaction is simulated using a real witness (rather than the "fake" witness). This can be done as the Real Execution Phase runs till the $i^{\text {th }}$ left interaction starts, and can also compute the real witness of the $i^{\text {th }}$ interaction. Note that these hybrids $\left\{\mathrm{hyb}^{i}\right\},\left\{\mathrm{hyb}_{+}^{i}\right\}$ are only concerned with producing a view of $A$, and do not extract the witnesses of the right interactions.

By construction, hyb ${ }^{i}$ and $\mathrm{hyb}_{+}^{i}$ abort only when $S$ aborts. Hence by Claim 2. we have,

Claim 4. For all $i, \mathrm{hyb}^{i}$ and hyb ${ }_{+}^{i}$ output $\perp$ with negligible probability.

By Claim 4, the output of hyb ${ }^{1}$ is statistically close to the output of $S$ running with $A$ in its entirety. (They only differ when $S$ aborts due to trying to extract witnesses of the right interactions from the $\mathrm{CECom}_{s b}$ 's committed by $A$.) The output of hyb ${ }^{m+1}$, on the other hand, is identical to the real view of $A$. Therefore Lemma 3 directly follows from the next two claims:

Lemma 5. The outputs of hyb $b_{+}^{i}$ and hyb ${ }^{i+1}$ are statistically close.

Proof. Ignoring the fact that hyb $b_{+}^{i}$ and hyb ${ }^{i+1}$ may abort, their outputs are identical. This is because $h_{y} b_{+}^{i}$ differs from hyb ${ }^{i+1}$ only in that when generating the output view, from the beginning of the $i^{\text {th }}$ left interaction until the beginning of the $i+1^{\text {st }}$ left interactions, hyb ${ }_{+}^{i}$ employs rewinds. However, these rewinds do not extract any new "fake witnesses" for use in the output view, and do not skew the output distribution because the rewinding schedule (including which rewind determines the output view) is oblivious. Since both machines abort at most with negligible probability by Claim 4, their outputs are statistically close.

Lemma 6. The outputs of $\mathrm{hyb}^{i}$ and $\mathrm{hyb}_{+}^{i}$ are computationally indistinguishable.

Proof. Assume for contradiction that there exists an adversary $A$ and a polynomial $p$, such that, for infinitely many $n \in N$, hyb ${ }^{i}$ and hyb ${ }_{+}^{i}$ are distinguishable with probability $1 / p(n)$. Towards reaching a contradiction, note that hyb ${ }^{i}$ and $\mathrm{hyb}_{+}^{i}$ differ only in how the $i^{\text {th }}$ left interaction is simulated (fake or real witness) in the rewindings. We thus want to violate the computational hiding property of Stage 2-4 of the protocol, or the strongly witness-indistinguishable property (implied by the $\mathcal{Z} \mathcal{K}$ property) of Stage 6 . However, two problems arise: (1) the 
Real Execution Phase of the two hybrids takes exponential steps, and (2) Stage 2-6 of the $i^{\text {th }}$ left interaction maybe rewound by the simulator $S$. Fix a $n \in N$ for which our hypothesis holds. To overcome the first problem, by our hypothesis, there must exist an execution of the Real Execution Phase - defined by the views of the adversary $\mathcal{V}_{A}$, the left prover $\mathcal{V}_{P}$ and the right verifier $\mathcal{V}_{V}$ produced in this phase - such that, conditioned on $\left(\mathcal{V}_{A}, \mathcal{V}_{P}, \mathcal{V}_{V}\right)$ occurring in the two hybrids, hyb ${ }^{i}$ and $\mathrm{hyb}_{+}^{i}$ are still distinguishable with probability $1 / p(n)$. Given $\left(\mathcal{V}_{A}, \mathcal{V}_{P}, \mathcal{V}_{V}\right)$, the rest of the hybrids (i.e., the Simulation Phase and Output Phase) can be generated efficiently.

Now it only remains to handle the second problem, that is, Stage 2-6 of the $i^{\text {th }}$ left interaction may be rewound in the Simulation Phase. We consider another two hybrids hyb $^{i}$ and hyb $_{+}^{i}$, which proceed identically to hyb ${ }^{i}$ and hyb ${ }_{+}^{i}$ respectively, except that, in the Simulation Phase, they employ the following alternative simulation strategy that avoids rewinding Stage 2-6 of the $i^{\text {th }}$ left interactions.

The Alternative Simulation Strategy of $\tilde{h y y b}^{i}$ : The goal of this simulation strategy is to complete the partial execution $\left(\mathcal{V}_{A}, \mathcal{V}_{P}, \mathcal{V}_{V}\right)$ produced by the Real Execution Phase, without rewinding Stage 2-6 of the $i^{\text {th }}$ left interaction. Let $\left\{m_{1}, \ldots, m_{t}\right\}$ for $t=k(n) / 2$, be the messages that $A$ sends in Stage 2-6 of the $i^{\text {th }}$ left interaction; and $a_{i}$ the reply to $m_{i}$ from the left prover. Then the execution of $A$ continuing from $\mathcal{V}_{A}$ is "equivalent" to the sequential execution of the following $t+1$ machines $A_{1}, \ldots, A_{t+1}$.

Machine $A_{i}$ on input a partial view $\mathcal{V}_{i-1}$ of $A$ up until the message $m_{i-1}$ is sent and the reply $a_{i-1}\left(\mathcal{V}_{0}=\mathcal{V}_{A}\right.$ and $\left.a_{0}=\varepsilon\right)$, continues the execution of $A$ from $\mathcal{V}_{i-1}$, by feeding $\mathcal{V}_{i-1}$ and $a_{i-1}$ to $A$, and forwarding every message from $A$ externally; finally, it aborts when $A$ terminates or sends the message $m_{i}$, and output the newly generated view $\mathcal{V}_{i}$ of $A$.

The alternative simulation strategy, instead of producing a simulated view of $A$ "in one shot", produces the view "progressively" by simulating the view of $A_{1}, \ldots, A_{t+1}$ in sequence. Furthermore, the simulation strategy remembers all the "fake witnesses" it has extracted so far, and to simulate the view of $A_{i}$, it can use the "fake" witnesses extracted when simulating the views of $A_{j}$ 's with $j<i$. More precisely, let $\mathcal{S}^{i}\left(S^{0}=\emptyset\right)$ denote the set of "fake witnesses" extracted after simulating the views of the first $i$ machines $A_{1}, \ldots, A_{i}$, and $\left(\mathcal{V}_{A}^{j}, \mathcal{V}_{P}^{j}, \mathcal{V}_{V}^{j}\right)$ $\left(\left(\mathcal{V}_{A}^{0}, \mathcal{V}_{P}^{0}, \mathcal{V}_{V}^{0}\right)=\left(\mathcal{V}_{A}, \mathcal{V}_{P}, \mathcal{V}_{V}\right)\right)$ the partial execution produced after simulating the first $i$ machines. In step $j \in[t+1]$,

1. Simulate the view of $A_{j}$ continuing from $\left(\mathcal{V}_{A}^{j-1}, \mathcal{V}_{P}^{j-1}, \mathcal{V}_{V}^{j-1}\right)$ as in hyb ${ }^{i}$ - that is, emulate the first $i-1$ left and all the right interactions honestly from $\mathcal{V}_{P}^{j-1}$ and $\mathcal{V}_{V}^{j-1}$, and simulate the rest $m-i+1$ left interactions using "fake" witnesses - except that now the "fake" witnesses can be obtained through extracting from some CECom ${ }_{s h}$ 's in this step, or in previous steps, found in $\mathcal{S}$. (Output $\perp_{\text {ext }}$ if no such fake witness is available, and $\perp_{\text {bind }}$, if $A_{i}$ decommits to a value different from any of the "fake" witnesses extracted.) 
2. Set $\mathcal{V}_{A}^{j}$ to the view of $A$ embedded in the simulated view of $A_{i}$ (set $\mathcal{V}_{P}^{j}$ and $\mathcal{V}_{V}^{j}$ appropriately as well); add all the "fake" witnesses extracted in this step to $\mathcal{S}$.

Finally, hỹb ${ }^{i}$ outputs $\mathcal{V}=\mathcal{V}_{A}^{t+1}$.

We remark that in step $j$, the only message that $A_{j}$ receives belonging to Stage $2-6$ of the $i^{\text {th }}$ left interaction is $a_{j-1}$. This is because $A$ in $A_{j}$ starts its execution from $\mathcal{V}_{A}^{j-1}$, after messages $m_{1}$ to $m_{j-1}$ are sent, and is cutoff immediately after $m_{j}$ is sent. Therefore, during the simulation with $A_{i}$, in every rewinding, $A$ never sends $m_{1}$ to $m_{j-1}$ again, and never receives a reply to $m_{j}$ (as every time it does send $m_{j}$, it is cutoff immediately). Hence the only message it receive is $a_{j-1}$. Therefore, overall, the alternative simulation strategy never rewinds Stage 2-6 of the $i^{\text {th }}$ left interaction.

The Alternative Simulation Strategy of hyb $_{+}^{i}$ : Define hyb $_{+}^{i}$ analogously for hyb ${ }_{+}^{i} \cdot \tilde{h y b}_{+}^{i}$ proceeds identically to $\tilde{h y y b}^{i}$, except that in the simulation with $A_{j}$ 's, messages in Stage 2-6 of the $i^{\text {th }}$ left interaction are emulated using the real witness (as in $\mathrm{hyb}_{+}^{i}$ ). As hy $\tilde{y y b}^{i}, \tilde{h y b}_{+}^{i}$ never rewinds Stage $2-6$ of the $i^{\text {th }}$ left interaction.

Claim 7. For all $i$, hyb $^{i}$ and hỹb $_{+}^{i}$ output $\perp$ with negligible probability.

Proof. It essentially follows from Claim 4 that the probabilities that hyb $^{i}$ and hyb outputs $\perp_{\text {bind }}$ are negligible.

On the other hand, $\tilde{h y b}^{i}$ (respectively $\tilde{h y b}_{+}^{i}$ ) outputs $\perp_{\text {ext }}$ only if it fails to extract a "fake" witness for some left interaction $j \geq i$ (respectively $j>i$ ). Fix one such $j$. Since left interaction $j$ starts completely after $\mathcal{V}_{A}$ (the view generated in the Real Execution Phase), the execution of this interaction occurs completely inside machines $A_{1}, \ldots, A_{t+1}$, where $t=k / 2$. Then since the number of CECom ${ }_{s h}$ 's in Stage 1 of the left interaction is $k+1>t+1$, there exists a machine $A_{j^{\prime}}$, such that, during its execution, a complete CECom sh from $A$ is sent. Then in Step $j^{\prime}$ of $\tilde{\tilde{y y b}}^{i}$ (respectively $\tilde{h y b}_{+}^{i}$ ), the alternative simulation strategy must try to extract a "fake" witness from this CECom , $_{\text {, }}$, and by Claim 4 , it succeeds except with negligible probability. Therefore, by union bound, the probability that $\tilde{\tilde{y b}}^{i}$ (respectively hy $\tilde{y}_{+}^{i}$ ) outputs $\perp_{\text {ext }}$ is negligible.

Furthermore, ignoring the fact that hyb $^{i}$ and hyb ${ }^{i}$ (resp., hỹb ${ }_{+}^{i}$ and hyb $_{+}^{i}$ ) may abort, their outputs are identical, since the views of $A$ in $\tilde{h y y b}^{i}$ and hyb ${ }^{i}$ are simulated identically. (This is because that the simulated view of $A$ depends only on the value of the "fake" witnesses extracted, and is otherwise oblivious of the extraction strategy. Following from the same proof as in Claim 2, we have that for every left interaction in which the adversary successfully decommits the Stage 1 commitment (in Stage 5), the "fake" witnesses extracted in the two hybrids are identical, except from negligible probability. For the rest left interactions, the extracted "fake" witnesses are never used in the simulation.) Therefore, 
Claim 8. For all $i$, it holds that the outputs of $\tilde{h y y b}^{i}$ and hyb ${ }^{i}$ are statistically close, and the outputs of $\mathrm{hyb}_{+}^{i}$ and $\mathrm{hyb}_{+}^{i}$ are statistically close.

Combining Claim 8 with our hypothesis, we have that conditioned on $\left(\mathcal{V}_{A}, \mathcal{V}_{P}, \mathcal{V}_{V}\right)$ occurring in the two hybrids, $\tilde{n y y b}^{i}$ and $\tilde{h y y b}_{+}^{i}$ are distinguishable with probability at least $1 / 2 p(n)$. Note that continuing from $\left(\mathcal{V}_{A}, \mathcal{V}_{P}, \mathcal{V}_{V}\right)$ the rest of the two hybrids can be efficiently generated, and the only difference between the two hybrids lies in how Stage 2-6 of the $i^{\text {th }}$ left interaction are simulated (using a fake or a real witness), which are never rewound in the two hybrids. Then it follows directly from the computational hiding property of Stage 2-4, and the strongly witness-indistinguishable property (implied by the $\mathcal{Z} \mathcal{K}$ property) of Stage 6 that conditioned on $\left(\mathcal{V}_{A}, \mathcal{V}_{P}, \mathcal{V}_{V}\right)$, $\tilde{h y b}^{i}$ and $\tilde{h y b}_{+}^{i}$ are indistinguishable. This gives a contradiction.

\section{Proof of $\mathcal{A C N \mathcal { M Z K }}$}

As shown in the last section, the simulator constructed in Section 4.1 is a correct $\mathcal{A C Z K}$ simulator; that is, the first output of $S$ (i.e., view $=S_{1}\left(1^{n}, z\right)$ ) is computationally indistinguishable from the real view of the adversary. To further

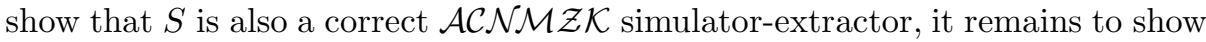
that the second output of $S$ contains the valid $\mathcal{N} \mathcal{P}$ witnesses of the statements proved in the right interactions (in view).

By construction, the witnesses that $S$ outputs are just values it extracts out from the CECom $s$ 's in Stage 2 of the right interactions. Therefore, if $A$ always commits to valid witnesses using $\mathrm{CECom}_{s b}$ in the right interactions, by Claim 2 the simulator $S$ would extract the valid witnesses except with negligible probability. Therefore, the following lemma establishes the correctness of the output witnesses:

Lemma 9. For every $\mathcal{P} \mathcal{P} \mathcal{T}$ adversary $A$, there exists a negligible function $\nu$, such that for every $n \in N$ and $z \in\{0,1\}^{*}$, the probability that $A$ fails to commit to a valid witness in Stage 2 of a right interaction that is accepting and uses a different identity from all left interactions in view $=S_{1}\left(1^{n}, z\right)$, is less than $\nu(n)$.

Proof. Assume for contradiction that there exists a man-in-the-middle adversary $A$ that participates in $m=m(n)$ left and right interactions, and a polynomial function $p$, such that for infinitely many $n \in N$ and $z \in\{0,1\}^{*}, A$ cheats in an outcome of $S_{1}\left(1^{n}, z\right)$ with probability $1 / p(n)$; by cheating, we mean that $A$ fails to commit to a valid witness in Stage 2 of any right interaction that is accepting and uses a different identity from all the left interactions. (Note that $A$ is not considered cheating if the simulator fails to output a view of $A$ ).

Consider again the series of hybrids, hyb ${ }^{i}$ and $h_{y b}{ }_{+}$, defined in section 4.2 . Since the output of hyb ${ }^{1}$ is statistically close to the output of $S$, by our hypothesis, the probability that $A$ cheats in hyb ${ }^{1}$ is non-negligible. On the other hand, in hyb ${ }^{m+1}$, it follows from the soundness of Stage 6 that, except with negligible probability, in every accepting right interaction, $A$ commits to either a real or a "fake" witness; it further follows from the statistically hiding property of Stage 
1 and the (stand-alone) extractability of Stage 2 that, except with negligible probability, $A$ never commits to a "fake" witness in any accepting right interactions. Hence, by union bound, except with negligible probability, $A$ never cheats in hyb ${ }^{m+1}$. It follows from Claim 6 that the probabilities of $A$ cheating in hyb ${ }_{+}^{i}$ and hyb ${ }^{i+1}$ differ by at most a negligible amount. Therefore, for infinitely many $n$, there must exist an $i=i(n)$, such that, the probabilities of $A$ cheating in hyb ${ }^{i}$ and $\mathrm{hyb}_{+}^{i}$ differ by at least a polynomial amount. Since the total number of right interactions is bounded by a polynomial, this implies that the probabilities that $A$ cheats in a randomly chosen right interaction in the two hybrids differ by a polynomial amount.

Notice that the hybrids hyb ${ }^{i}$ and hyb $b_{+}^{i}$ proceed identically up until the $i^{\text {th }}$ left interaction starts. After that, the only difference between the two experiments lies in how the $i^{\text {th }}$ left interaction is simulated (using either the fake or real witnesses). Towards reaching a contradiction, we want to claim that, by the non-malleability and $\ell(n)$-robustness of NMCom, the value $A$ commits to in a randomly chosen right interaction is "computationally independent" from how Stage 2-6 of the $i^{\text {th }}$ left interaction are simulated. However, (as in proof of Lemma 5) two problems arise: one is that the Real Execution Phase of the two hybrids can not be generated efficiently, and the other is that both Stage $2-6$ of the $i^{\text {th }}$ left and the randomly chosen right interactions might be rewound by $S$. We solve the two problem in the same way as in proof of Lemma 5 , to overcome the first problem, we fix one execution of the Real Execution Phase $\left(\mathcal{V}_{A}, \mathcal{V}_{P}, \mathcal{V}_{V}\right)$ such that conditioned on it occurring, the two hybrids are still distinguishable with high probability; to overcome the second problem, we again consider two alternative hybrids hyb $\hat{~}^{i}$ and $\hat{h y b}_{+}{ }^{i}$, which proceed identically to hyb ${ }^{i}$ and $\mathrm{hyb}_{+}^{i}$ respectively, except that, in the Simulation Phase, they employ an alternative simulation strategy that avoids rewinding Stage 2-6 of the $i^{\text {th }}$ left and the randomly picked right interactions. More precisely, hyb $\hat{b}^{i}$ and $\hat{h y b}_{+}^{i}$ proceed almost identically to $\tilde{h y y b}^{i}$ and $\tilde{h y b}_{+}^{i}$ in the proof of Lemma 5 , except that now it "chops" up the execution of $A$ into $k(n)+1$ phases $A_{1}, \ldots, A_{k+1}$, according to messages in Stage 2-6 of the $i^{\text {th }}$ left and the randomly picked right interactions, and simulates the views of $A_{1}, \ldots, A_{k+1}$ sequentially. It follows using the same argument that the outputs of $\hat{h y b}^{i}$ and $\mathrm{hyb}^{i}$, as well as that of $\hat{h y b}_{+}^{i}$ and hyb $_{+}^{i}$, are statistically close.

Therefore by our hypothesis, the probabilities that $A$ cheats in a randomly chosen right interaction in $\hat{h y b}^{i}$ and $\hat{h y b}_{+}^{i}$ differ by a polynomial amount. However, the only difference between the two hybrids lies in how Stage 2-6 of the $i^{\text {th }}$ left interaction are simulated (using a fake or a real witness), and the Stage 2-6 of the $i^{\text {th }}$ left and the randomly chosen right interactions are never rewound in the two hybrids. Then it follows using the same proof of Lemma 7 in LPTV10] that, essentially by the non-malleability and $\ell(n)$-robustness of NMCom that the probability that $A$ commits to a "fake" witness in Stage 2 of the randomly chosen right interaction differ by at most a negligible amount, which gives a contradiction. 
Acknowledgement. We thank Vipul Goyal and the anonymous referees of TCC 2011 for many helpful comments and inspiring suggestions.

\section{References}

[BCC88] Brassard, G., Chaum, D., Crépeau, C.: Minimum disclosure proofs of knowledge. J. Comput. Syst. Sci. 37(2), 156-189 (1988)

[Blu86] Blum, M.: How to prove a theorem so no one else can claim it. In: Proc. of the International Congress of Mathematicians, pp. 1444-1451 (1986)

[BPS06] Barak, B., Prabhakaran, M., Sahai, A.: Concurrent non-malleable zero knowledge. In: FOCS, pp. 345-354 (2006)

[Can01] Canetti, R.: Universally composable security: A new paradigm for cryptographic protocols. In: FOCS 2001: Proceedings of the 42nd IEEE Symposium on Foundations of Computer Science, Washington, DC, USA, p. 136. IEEE Computer Society, Los Alamitos (2001)

[CF01] Canetti, R., Fischlin, M.: Universally Composable Commitments. In: Kilian, J. (ed.) CRYPTO 2001. LNCS, vol. 2139, pp. 19-40. Springer, Heidelberg (2001)

[CKL03] Canetti, R., Kushilevitz, E., Lindell, Y.: On the limitations of universally composable two-party computation without set-up assumptions. In: Biham, E. (ed.) EUROCRYPT 2003. LNCS, vol. 2656, pp. 68-86. Springer, Heidelberg (2003)

[DDN00] Dolev, D., Dwork, C., Naor, M.: Nonmalleable cryptography. SIAM Journal on Computing 30(2), 391-437 (2000)

[DN02] Damgård, I., Nielsen, J.B.: Perfect hiding and perfect binding universally composable commitment schemes with constant expansion factor. In: Yung, M. (ed.) CRYPTO 2002. LNCS, vol. 2442, pp. 581-596. Springer, Heidelberg (2002)

[DNS04] Dwork, C., Naor, M., Sahai, A.: Concurrent zero-knowledge. J. ACM 51(6), 851-898 (2004)

[GK96] Goldreich, O., Kahan, A.: How to construct constant-round zeroknowledge proof systems for NP. Journal of Cryptology 9(3), 167-190 (1996)

[GMR89] Goldwasser, S., Micali, S., Rackoff, C.: The knowledge complexity of interactive proof systems. SIAM Journal on Computing 18(1), 186-208 (1989)

[GMW91] Goldreich, O., Micali, S., Wigderson, A.: Proofs that yield nothing but their validity or all languages in NP have zero-knowledge proof systems. J. ACM 38(3), 690-728 (1991)

[Gol01] Goldreich, O.: Foundations of Cryptography — Basic Tools. Cambridge University Press, Cambridge (2001)

[KP01] Kilian, J., Petrank, E.: Concurrent and resettable zero-knowledge in poly-loalgorithm rounds. In: STOC 2001, pp. 560-569 (2001)

[Lin03] Lindell, Y.: Bounded-concurrent secure two-party computation without setup assumptions. In: STOC 2003, pp. 683-692 (2003)

[LP09] Lin, H., Pass, R.: Non-malleability amplification. In: STOC 2009, pp. 189-198 (2009)

[LPTV10] Lin, H., Pass, R., Tseng, W.-L.D., Venkitasubramaniam, M.: Concurrent non-malleable zero knowledge proofs. In: Rabin, T. (ed.) CRYPTO 2010. LNCS, vol. 6223, pp. 429-446. Springer, Heidelberg (2010) 
[LPV08] Lin, H., Pass, R., Venkitasubramaniam, M.: Concurrent Non-malleable Commitments from Any One-Way Function. In: Canetti, R. (ed.) TCC 2008. LNCS, vol. 4948, pp. 571-588. Springer, Heidelberg (2008)

[MOSV06] Micciancio, D., Ong, S.J., Sahai, A., Vadhan, S.: Concurrent Zero Knowledge Without Complexity Assumptions. In: Halevi, S., Rabin, T. (eds.) TCC 2006. LNCS, vol. 3876, pp. 1-20. Springer, Heidelberg (2006)

[OPV10] Ostrovsky, R., Pandey, O., Visconti, I.: Efficiency preserving transformations for concurrent non-malleable zero knowledge. In: Micciancio, D. (ed.) TCC 2010. LNCS, vol. 5978, pp. 535-552. Springer, Heidelberg (2010)

[PR05] Pass, R., Rosen, A.: New and improved constructions of non-malleable cryptographic protocols. In: STOC 2005, pp. 533-542 (2005)

[PRS02] Prabhakaran, M., Rosen, A., Sahai, A.: Concurrent zero knowledge with logarithmic round-complexity. In: FOCS 2002, pp. 366-375 (2002)

[PTV08] Pass, R., Tseng, W.-L.D., Venkitasubramaniam, M.: Concurrent zero knowledge: Simplifications and generalizations(2008) (manuscript), http://hdl.handle.net/1813/10772

$\left[\mathrm{SCO}^{+} 01\right]$ De Santis, A., Di Crescenzo, G., Ostrovsky, R., Persiano, G., Sahai, A.: Robust non-interactive zero knowledge. In: Kilian, J. (ed.) CRYPTO 2001. LNCS, vol. 2139, pp. 566-598. Springer, Heidelberg (2001)

[YYZ09] Yao, A.C.-C., Yung, M., Zhao, Y.: Adaptive concurrent non-malleability with bare public-keys. CoRR, abs/0910.3282 (2009) 